\title{
Erratum: General polarizability and hyperpolarizability estimators for the path-integral Monte Carlo method applied to small atoms, ions, and molecules at finite temperatures \\ [Phys. Rev. A 94, 032515 (2016)]
}

Juha Tiihonen, Ilkka Kylänpää, and Tapio T. Rantala

(Received 7 July 2017; published 19 July 2017)

DOI: 10.1103/PhysRevA.96.019902

In our recent work we derived field-free estimators for static polarizabilities and hyperpolarizabilities for the path-integral Monte Carlo method. Our derivation contained some unconventional practices of notation and sign. For example, we had replaced $F_{\alpha} F_{\beta}$ with $F_{\alpha \beta}$, etc., whereas the latter is commonly used for a field gradient, i.e., $F_{\alpha \beta}=\left(\nabla F_{\alpha}\right)_{\beta}$. Also, in Eqs. (5)-(9) there were some sign errors in contrast to Ref. [1]. However, these signs cancel out in either case, leaving the resulting estimators and the data unaffected. The correct way to write the equations would be the following:

$$
\begin{gathered}
E^{(1)}=E^{(0)}-\mu_{\alpha} F_{\alpha}-\frac{1}{2} \alpha_{\alpha \beta} F_{\alpha} F_{\beta}-\frac{1}{6} \beta_{\alpha \beta \gamma} F_{\alpha} F_{\beta} F_{\gamma}-\frac{1}{24} \gamma_{\alpha \beta \gamma \delta} F_{\alpha} F_{\beta} F_{\gamma} F_{\delta}-\cdots, \\
\mu_{\alpha}=-\lim _{F \rightarrow 0} \frac{\partial}{\partial F_{\alpha}} E^{(1)}, \\
\alpha_{\alpha \beta}=-\lim _{F \rightarrow 0} \frac{\partial}{\partial F_{\alpha}} \frac{\partial}{\partial F_{\beta}} E^{(1)}=\lim _{F \rightarrow 0} \frac{\partial}{\partial F_{\beta}} \mu_{\alpha}, \\
\beta_{\alpha \beta \gamma}=-\lim _{F \rightarrow 0} \frac{\partial}{\partial F_{\alpha}} \frac{\partial}{\partial F_{\beta}} \frac{\partial}{\partial F_{\gamma}} E^{(1)}=\lim _{F \rightarrow 0} \frac{\partial}{\partial F_{\gamma}} \alpha_{\alpha \beta}, \\
\gamma_{\alpha \beta \gamma \delta}=-\lim _{F \rightarrow 0} \frac{\partial}{\partial F_{\alpha}} \frac{\partial}{\partial F_{\beta}} \frac{\partial}{\partial F_{\gamma}} \frac{\partial}{\partial F_{\delta}} E^{(1)}=\lim _{F \rightarrow 0} \frac{\partial}{\partial F_{\delta}} \beta_{\alpha \beta \gamma} .
\end{gathered}
$$

Furthermore, we want to publish one more nonvanishing property that was left out in the original article. Namely, $\beta_{y y y}=1.12(14)$ for $\mathrm{H}_{3}^{+}$in the adiabatic (fixed-nuclei) simulation.

[1] A. D. Buckingham, in Advances in Chemical Physics: Intermolecular Forces, edited by J. O. Hirschfelder, Advances in Chemical Physics Vol. 12 (Wiley, Hoboken, NJ, 2007), pp. 107-142. 